\title{
Constitutively active SMAD2/3 facilitates efficient transcription factor-mediated cell conversion
}

\author{
Sophia Kelaini, Andriana Margariti
}

The Wellcome-Wolfson Institute for Experimental Medicine, School of Medicine, Dentistry and Biomedical Sciences, Queen's University Belfast, Belfast, UK

Correspondence to: Dr. Andriana Margariti, PhD. Centre for Experimental Medicine, Queen's University Belfast, Wellcome-Wolfson Building, 97 Lisburn Road, BT9 7BL, UK. Email: a.margariti@qub.ac.uk.

Comment on: Ruetz T, Pfisterer U, Di Stefano B, et al. Constitutively Active SMAD2/3 Are Broad-Scope Potentiators of Transcription-FactorMediated Cellular Reprogramming. Cell Stem Cell 2017;21:791-805.e9.

Received: 12 July 2018; Accepted: 19 July 2018; Published: 22 August 2018.

doi: $10.21037 /$ sci.2018.08.03

View this article at: http://dx.doi.org/10.21037/sci.2018.08.03

The generation of induced pluripotent stem cells (iPSCs) is a promising and exciting tool for regenerative medicine. Since their first appearance in 2006 by Yamanaka et al, where adult mouse fibroblasts were reprogrammed using a cocktail of only four master transcription factors (TFs), Oct4, Sox2, Klf4, and $c-M y c$ reprogramming factors (1), iPSCs have served as valuable tools in studying disease. Indeed, even years after this groundbreaking discovery, cellular identity conversion of somatic cells through exogenous introduction of TFs towards iPSCs remains one of the most powerful tools (2) for disease modelling, drug screening, tissue engineering and transplantation therapies. Nevertheless, despite recent advances, the induction efficiency of these cells and subsequent differentiation to the desired cell types is, very low (3) and has a lengthy reprogramming process with substantial limitations to create and maintain functional cells. In a combined effort, laboratories led by Keisuke Kaji investigated a new approach of improving the efficiency of iPSCs generation by the reprogramming factors through the expression of constitutively active SMAD2/3 (4).

Several cell conversion approaches (5) mediated by TFs have been reported to improve cell reprogramming, such as the addition of small molecules (6) involved in epigenetic or signalling pathways, which can even replace one or more reprogramming factors, including Sox2 or $c-M y c$ (7). Since transforming growth factor $\beta$ (TGF- $\beta$ ) signalling counteracts the mesenchymal to epithelial transition (MET) (8), a crucial event for iPSCs generation from fibroblasts (9), transforming growth factor $\beta$ receptor (TGF- $\beta$ R) inhibitors have, thus, been shown to enhance the reprogramming process. The TGF- $\beta$ signalling pathway is involved in a large number of cellular processes and includes the SMAD proteins, which, after activation by receptors, translocate into the nucleus to act as TFs and regulate transcription (10). SMAD2/3 also interact with other TFs as well as transcriptional coactivators, nucleosome modifiers (11) and chromatin remodelers (12).

In the study by Kaji et al. treatment with TGF$\beta R$ inhibitors reduced apoptosis in the early stages of reprogramming by suppressing reprogramming-dependent p19ARF upregulation, a p53-dependent cell cycle arrest and senescence inducer. In particular, to investigate how TGF$\beta R$ inhibitors may enhance reprogramming, the researchers supplemented the reprogramming media with A83-01 (A83) which is an inhibitor of the TGF- $\beta$-induced epithelialto-mesenchymal transition (EMT). This led to a 3 -fold increase in reprogramming efficiency with more Nanogpositive colonies in the presence of A83. Other than the reduction in apoptosis at the early stages of reprogramming, their analysis also revealed that reprogramming was strongly accelerated after addition of A83 treatment, including late reprogramming intermediates. This part of the investigation established that TGF- $\beta$ inhibition can positively influence colony formation even post-MET.

Continuing their investigation, when they prolonged the inhibitory treatment, SMAD2/3 showed increased levels in cultured cells. This led to the researchers assessing the effect of a constitutively active SMAD2/3 (Smad2/3CA) on the reprogramming process and subsequently identifying a 
clear enhancement in reprogramming speed and efficiency. Taking a closer look at these observations, it was revealed that the majority of the generated Nanog-positive cells are also positive for phosphorylated SMAD3 (p-SMAD3+), the active form of SMAD3, and TGF- $\beta$ R inhibitor treatment increased its occurrence in the cells. The follow up question was therefore "do constitutively active forms of SMAD2 (SMAD2CA) or SMAD3 (SMAD3CA) improve reprogramming?" Interestingly, overexpression of Smad2CA and/or Smad3CA led to increased numbers of Nanog-positive colonies. Alternatively, Smad2/3 double knockout (KO) in the presence or absence of A83 did not have any apparent effects on reprogramming. This signified that reprogramming enhancement by $\mathrm{A} 83$ was in general not dependent on endogenous SMAD2/3 but largely on increased exogenous SMAD2/3 levels. Global gene expression profiling, in particular, revealed that even though SMAD3CA-positive cells were not significantly different to embryonic stem cells (ESCs), they demonstrated higher expression of genes linked to pluripotency. This indicated that SMAD3CA followed the same general gene expression pattern as the one brought about by the reprogramming factors, but in an enhanced and accelerated fashion.

Further research by the groups also revealed that, during reprogramming to iPSCs, overexpressed SMAD3 was involved in a physical interaction with reprogramming factors and co-occupied OCT4 binding sites within the genome, possibly facilitating recruitment of transcriptional activators and chromatin remodelers, and, thus, enhancing conversion. More specifically, they showed that SMAD3 acts as a bridge between reprogramming factors, such as OCT4, and co-activators. During reprogramming, they observed that binding sites for Oct4 were occupied in parallel with SMAD3; however, no binding was detected in the absence of Yamanaka factors expression. Interestingly, SMAD3CA, known to interact with histone modifiers, may contribute to chromatin remodelling and histone changes during reprogramming, assisting, thus, in easier cell conversion to iPSCs.

Furthermore, active SMAD3 was shown to enhance cell conversions mediated by more master-TFs. More specifically, since SMAD3 is reported to interact with many different cell specific master TFs (13), the researchers looked to identify whether SMAD2/3CA could improve more TF-mediated cell conversions. For example, when B cells were converted to macrophages by overexpressing Cebpa (14), 75\% of Smad2/3CA expressing cells expressed the macrophage marker MAC- 1 on day 4 after conversion, compared to less than $30 \%$ of cells converted with only Cebpa. These results showed that SMAD2/3CA significantly boosted $\mathrm{B}$ cell to macrophage conversion mediated by $\mathrm{CEBP} \alpha$, possibly by facilitating its recruitment to corresponding CEBP $\alpha$ targets (15). Next, myoblasts were converted to adipocytes using $\operatorname{Cebp} \beta$ and $\operatorname{Prdm} 16$. Co-expression with Smad2CA or Smad3CA increased cell conversion efficiency. Similarly, human fibroblasts were converted to neurons with Ascl1, Brn2a, MytIl, and NeuroD1. They observed that SMAD3CA expression sped up alterations in gene expression universally, in a similar fashion to that of iPSCs production.

Starting with an investigation of TGF- $\beta$ R inhibitors and how they may enhance reprogramming efficiency, this study, identified that exogenous, constitutive active SMAD2/3 is an important factor in successful cell conversion. This observation was made not only in iPSCs generation but also in other TF-mediated cell fate changes suggesting a crucial role of these factors in regenerative medicine, where swift and efficient generation of functional mature cells is a great necessity. These findings by Kaji et al. can, thus, provide us with another powerful tool to overcome cell conversion challenges and help us bypass the barriers in the road towards more efficient cell reprogramming.

\section{Acknowledgements}

This work is funded by BHF and BBSRC.

\section{Footnote}

Conflicts of Interest: The authors have no conflicts of interest to declare.

\section{References}

1. Takahashi K, Yamanaka S. Induction of Pluripotent Stem Cells from Mouse Embryonic and Adult Fibroblast Cultures by Defined Factors. Cell 2006;126:663-76.

2. Lee G, Papapetrou EP, Kim H, et al. Modelling pathogenesis and treatment of familial dysautonomia using patient-specific iPSCs. Nature 2009;461:402-6.

3. Kogut I, McCarthy SM, Pavlova M, et al. High-efficiency RNA-based reprogramming of human primary fibroblasts. Nat Commun 2018;9:745.

4. Ruetz T, Pfisterer U, Di Stefano B, et al. Constitutively Active SMAD2/3 Are Broad-Scope Potentiators of Transcription-Factor-Mediated Cellular Reprogramming. 
Cell Stem Cell 2017;21:791-805 e9.

5. Cochrane A, Kelaini S, Tsifaki M, et al. Quaking Is a Key Regulator of Endothelial Cell Differentiation, Neovascularization, and Angiogenesis. Stem Cells (Dayton, Ohio) 2017;35:952-66.

6. Wang H, Cao N, Spencer CI, et al. Small molecules enable cardiac reprogramming of mouse fibroblasts with a single factor, Oct4. Cell Rep 2014;6:951-60.

7. Maherali N, Hochedlinger K. Tgfbeta signal inhibition cooperates in the induction of iPSCs and replaces Sox 2 and cMyc. Curr Biol 2009;19:1718-23.

8. Xu J, Liu X, Jiang Y, et al. MAPK/ERK signalling mediates VEGF-induced bone marrow stem cell differentiation into endothelial cell. J Cell Mol Med 2008;12:2395-406.

9. Li R, Liang J, Ni S, et al. A mesenchymal-to-epithelial transition initiates and is required for the nuclear reprogramming of mouse fibroblasts. Cell Stem Cell 2010;7:51-63.

10. Derynck R, Zhang YE. Smad-dependent and Smad-

doi: $10.21037 /$ sci.2018.08.03

Cite this article as: Kelaini S, Margariti A. Constitutively active SMAD2/3 facilitates efficient transcription factormediated cell conversion. Stem Cell Investig 2018;5:25. independent pathways in TGF-beta family signalling. Nature 2003;425:577-84.

11. Feng XH, Zhang Y, Wu RY, et al. The tumor suppressor Smad4/DPC4 and transcriptional adaptor CBP/ p300 are coactivators for smad3 in TGF-beta-induced transcriptional activation. Genes Dev 1998;12:2153-63.

12. Xi Q, He W, Zhang XH, et al. Genome-wide impact of the BRG1 SWI/SNF chromatin remodeler on the transforming growth factor beta transcriptional program. J Biol Chem 2008;283:1146-55.

13. Massague J. TGFbeta signalling in context. Nat Rev Mol Cell Biol 2012;13:616-30.

14. Fukuchi Y, Shibata F, Ito M, et al. Comprehensive analysis of myeloid lineage conversion using mice expressing an inducible form of C/EBP alpha. EMBO J 2006;25:3398-410.

15. Di Stefano B, Collombet S, Jakobsen JS, et al. C/EBPalpha creates elite cells for iPSC reprogramming by upregulating Klf4 and increasing the levels of Lsd1 and Brd4. Nat Cell Biol 2016;18:371-81. 